\title{
ASYMPTOTIC CLASSIFICATION OF ALIASING STRUCTURES ${ }^{1}$
}

\author{
R.N. IZMAILOV and A.V. POKROVSKII \\ Institute for Information Transmission Problems \\ Ermolovoy str., 19 \\ Moscow, 101447 RUSSIA
}

\begin{abstract}
The effects of quantization of quickly oscillating functions are considered. An asymptotical classification of aliasing spots is considered. The results obtained may be used in the restoring of certain features of initial functions.
\end{abstract}

Key words: Quantization, aliasing, computer graphics, lattice.

AMS (MOS) subject classifications: $\quad 68 \mathrm{U} 05,68 \mathrm{U} 10,06 \mathrm{Bxx}$.

\section{INTRODUCTION}

Consider a smooth function $F=F(x, y)$, where $(x, y) \in P=\vdash^{2}$. To make a qualitative description of the function $F$ the following method is convenient. Fix a number $m \in t$ and multiply the value of $F$ in each point $(x, y)$ by $\mathrm{m}$. If the integer approximation of $\mathrm{m} F(x, y)$ is odd (even) then the point $(x, y)$ is painted by white (black). So the plane $P$, painted black and white, graphically describes the behavior of the function $F$.

Now consider the restriction of this picture on the rectangular lattice $P \subseteq P$. For example, the computer monitor may be considered as a part of such a lattice. Of course, the quantization sharply changes the qualitative pictures and new structures appear.

Such structures are well known in the computer graphics: the quantization of oscillating objects may generate such parasite artifacts (aliasing). This problem was studied in recent years not only in special monographs [4] and [5] but also in popular magazines like [1-3]. The classification and description of the aliasing structures seem to be important not only for computer graphics but also for information transmission problems, etc. The analysis of such structures may be also useful in order to extract information on the quantization lattice and the initial function $F$.

\footnotetext{
${ }^{1}$ Received: November, 1991. Revised: June, 1992.
} 


\section{BASIC DEFINITIONS}

Fix a smooth function $F=F(x, y)$ defined on the plane $P=\mathbb{R}^{2}$. Denote by $L(n ; F, \mu)$ the following level set of the function $F$ :

$$
L(n ; F, \mu)=\{(x, y) \in P \text { such that } n-1 / 2<\mu F(x, y)<n+1 / 2\}
$$

where $\mu \in \mathbb{R}, n \in \mathbb{Z}$. For any $q \in \mathbb{Z}$ define the sets

$$
L_{q}(\nu ; F, \mu)=\bigcup_{k \equiv \nu \bmod q} L(k ; F, \mu),
$$

where $\nu=0,1, \ldots, q-1$. Consider a rectangular lattice $P(h, \alpha) \subseteq P$ with small steps $h_{x}=h \alpha$ and $h_{y}=h \alpha^{-1}$ along the axes $x$ and $y$, where the positive number $\alpha$ is fixed. The aim of the paper is to describe the quantized level sets

$$
L_{q}(\nu ; F, h, \alpha, \mu)=L_{q}(\nu ; F, \mu) \cap P(h, \alpha) .
$$

For each node $\left(x_{0}, y_{0}\right)$ of the lattice $\boldsymbol{P}(h, \alpha)$ define the rectangle

$$
Q\left(x_{0}, y_{0}\right)=\left\{(x, y) \in P:\left|x-x_{0}\right|<h_{x} / 2,\left|y-y_{0}\right|<h_{y} / 2\right\} .
$$

For each subset $M \subseteq P(h, \alpha)$ define the set

$$
Q(\boldsymbol{M})=\bigcup_{(x, y) \in M Q(x, y)} .
$$

Definition 1: $\quad$ Let $\chi \geq 0$. The measurable set $M \subseteq P$ with finite positive Lebesgue measure mes is said to be $\chi$-representable by the set $M \subseteq P$ if

$$
\frac{\operatorname{mes} \Delta(Q(M), M)}{\operatorname{mes}(M)}<\chi
$$

where $\Delta$ denotes the symmetric difference of sets.

If $h \rightarrow 0$ while $\mu=$ const, then the sets $L_{q}(\nu ; F, \mu)$ are $\chi$-representable by the sets $\boldsymbol{L}_{q}(\nu ; F, h, \alpha, \mu)$ with $\chi \rightarrow 0$. But this is not true if $\mu$ increases rapidly while $h$ vanishes. Furthermore, the cases

$$
h \rightarrow 0, \mu \rightarrow 0, \text { where } \mu h \equiv \Xi
$$

for a fixed $\Xi$ will be considered.

Definition 2: $\quad$ The sets $L_{q}(\nu ; F, h, \alpha, \Xi / h)$ asymptotically represent the function $G(x, y)$ in an open bounded $\Omega \subseteq P$ if for each small $h$ there exist $\xi=\xi(h)$ and $\chi=\chi(h)$ such that the sets

$$
L_{q}(\nu ; G(x, y)+\xi(h), \Xi / h) \cap \Omega
$$


are $\chi$-representable by the sets

$$
L_{q}(\nu ; F, h, \alpha, \Xi / h) \cap \Omega
$$

for each $n=0,1, \ldots, q-1$, where $\chi \rightarrow 0$ while $h \rightarrow 0$.

\section{EXAMPLES}

Example 1: $\quad$ Consider the function

$$
F_{1}(x, y)=-y^{4}+x^{2} y, \text { where }-1 \leq x, y \leq 1 .
$$

This function will be quantized by the lattice generated by a computer screen which consists of $641 \times 321$ points. So each pixel of the screen is a rectangle with sides $h_{x}=1 / 320, h_{y}=1 / 160$ :

$$
h=\frac{\sqrt{2}}{320}, \alpha=\sqrt{2} .
$$

In Fig. 1: the set $\boldsymbol{L}_{q}(0 ; F, h, \alpha, \mu)$ is drawn with white and the set $\boldsymbol{L}_{q}(1 ; F, h, \alpha, \mu)$ is drawn with black, where $q=2$ and $\mu=900$.

Example 2: $\quad$ The function in the previous example is the first function that the authors considered. The function

$$
F_{2}(x, y)=R-\sqrt{R^{2}-x^{2}-y^{2}}, \text { where }-1 \leq x, y \leq 1
$$

corresponds to the interference picture (Newton rings) generated with a monochrome light beam (with wave length $\mu$ ) and the lens of radius $R$ which lies on the flat discrete surface $P$ and touches it at the zero point. The aliasing effects for $\mu=2000$ are shown in Fig. 2; such effects might be seen when Newton rings are visualized by the digital screen.

\section{RESULTS}

Define the set

$$
D(F, \Xi)=\left\{d \in P \mid F_{x}^{\prime}(d)=\left(i q \Xi^{-1}\right) \alpha^{-1}, F_{y}^{\prime}(d)=\left(j q \Xi^{-1}\right) \alpha, i, j, \in \mathbb{Z}\right\} .
$$

For each point $d=\left(d_{x}, d_{y}\right) \in D(F, \Xi)$ define the function

$$
G_{d}(x, y)=F(x, y)-F_{x}^{\prime}(d)\left(x-d_{x}\right)-F_{y}^{\prime}\left(y-d_{y}\right)
$$

Theorem 1: For each point $d \in D(F, \Xi)$ the sets $L_{1}(\nu ; F, h, \alpha, \Xi / h)$ asymptotically represent the function $G_{d}(x, y)$. 
Proof: Let

$$
\begin{aligned}
& d_{x}=N_{x} h_{x}+\epsilon_{x}, x=\left(N_{x}+n_{x}\right) h_{x}, \\
& d_{y}=N_{y} h_{y}+\epsilon_{y}, y=\left(N_{y}+n_{y}\right) h_{y},
\end{aligned}
$$

where $N_{x}, N_{y}, n_{x}, n_{y} \in \mathbb{Z}$ and

$$
0 \leq \epsilon_{x}<h_{x}, 0 \leq \epsilon_{y}<h_{y}
$$

Suppose also that

$$
F_{x}^{\prime}(d)=\left(i q \Xi^{-1}\right) \alpha^{-1}, F_{y}^{\prime}(d)=\left(j q \Xi^{-1}\right) \alpha,
$$

where $i, j \in \mathbb{Z}$. Then the difference $\mu \nabla F$ between the functions $\mu F$ and $\mu G_{d}$ takes the form

$$
\begin{gathered}
\mu \nabla F=\mu\left(F_{x}^{\prime}(d)\left(x-d_{x}\right)-F_{y}^{\prime}\left(y-d_{y}\right)\right) \\
=\equiv\left(\frac{h i q}{\Xi h_{x}}\left(n_{x}-\epsilon_{x}\right)+\frac{h j q}{\Xi h_{y}}\left(n_{y}-\epsilon_{y}\right)\right) .
\end{gathered}
$$

Hence,

$$
\begin{aligned}
\mu \nabla F= & q\left(i n_{x}+j n_{y}\right)-q\left(i \epsilon h_{x}^{-1}+j \epsilon_{y} / h_{y}^{-1}\right) \\
& \equiv-q\left(i \epsilon h_{x}+j \epsilon_{y} h_{y}\right) \bmod q .
\end{aligned}
$$

Now the statement of the theorem follows from Definition 2 with

$$
\xi(h)=-q\left(i \epsilon h_{x}^{-1}+j \epsilon_{y} h_{y}^{-1}\right) .
$$

Corollary 1: $\quad$ Let the matrix $F^{\prime \prime}(d)$ be invertible at a point $d=\left(d_{x}, d_{y}\right) \in D(F, \Xi)$. Then there exists a positive $r$ such that the sets $L_{q}\left(\nu ; F, h, \alpha, \Xi h^{-1}\right)$ asymptotically represent the bilinear function

$$
H(x, y)=\left(F^{\prime \prime}(d)\left(x-d_{x}, y-d_{y}\right),\left(x-d_{x}, y-d_{y}\right)\right)
$$

in the circle

$$
B_{r}(d)=\left\{(x, y) \in P||\left(x-d_{x}\right)^{2}+\left(y-d_{y}\right)^{2} \mid<r^{2}\right\}
$$

Proof follows from Theorem 1 and the Taylor expansion of $F$ in $B_{r}(d)$.

Hence, the forms of the main aliasing spots are defined by the signature of the matrix $F^{\prime \prime}(d)$. Each point of $D(F, \Xi)$ coincides with the center of the main aliasing spot (the error is less than a pixel).

Suppose that in a sufficiently large domain $\Omega$ the matrix $F^{\prime \prime}(x, y)$ is invertible and varies 
slowly:

$$
\sup _{d \in \Omega}\left|F^{\prime \prime \prime}(d)\right| \leq \varphi
$$

where $\varphi$ is small. Then the aliasing spots form a curved lattice which is generated by the curves

$$
\begin{gathered}
C_{x}(i, F)=\left\{(x, y) \in P \mid F_{x}^{\prime}(x, y)=\left(i q \Xi^{-1}\right) \alpha^{-1},\right\} \\
C_{y}(j, F)=\left\{(x, y) \in P \mid F_{y}^{\prime}(x, y)=\left(j q \Xi^{-1}\right) \alpha ;\right\} .
\end{gathered}
$$

The set $D(F, \Xi)$ is generated by the intersections $d(i, j ; F, \Xi)$ of the curves $C_{x}(i, F)$ and $C_{y}(j, F)$. Define the vector steps $X, Y$ of this lattice as

$$
\begin{aligned}
& X^{i j}=\left(X_{x}^{i j}, X_{y}^{i j}\right)=d(i+1, j ; F, \Xi)-d(i, j ; F, \Xi) \\
& Y^{i j}=\left(Y_{x}^{i j}, Y_{y}^{i j}\right)=d(i, j+1 ; F, \Xi)-d(i, j ; F, \Xi) .
\end{aligned}
$$

Note that since the points $d(i, j ; F, \Xi)$ do not change as $h \rightarrow 0$, the vectors $X$ and $Y$ also do not change.

Theorem 2:

$$
\lim _{\varphi \rightarrow 0} \alpha \sqrt{\frac{X_{y}}{Y_{x}}}=1 .
$$

Proof: Consider the Taylor expansion of the functions $F_{x}^{\prime}$ and $F_{y}^{\prime}$ in the neighborhood $\Omega$ of the point $d^{*}=d(i, j ; F, \Xi)$. Then the definitions of $X, Y$ and $d(i, j ; F, \Xi)$ imply the relations

$$
\begin{aligned}
& \frac{q}{\Xi \alpha} F^{\prime \prime}\left(d^{*}\right) X \simeq\left(\begin{array}{l}
1 \\
0
\end{array}\right) \\
& \frac{q \alpha}{\Xi} F^{\prime \prime}\left(d^{*}\right) Y \simeq\left(\begin{array}{l}
0 \\
1
\end{array}\right)
\end{aligned}
$$

here $\simeq$ denotes the first order of approximation as $h \rightarrow 0$ and $\varphi \rightarrow 0$. Hence

$$
\begin{aligned}
& \frac{\alpha \Xi}{q}\left(F^{\prime \prime}\left(d^{*}\right)\right)^{-1}\left(\begin{array}{l}
1 \\
0
\end{array}\right) \simeq X, \\
& \frac{\Xi}{\alpha q}\left(F^{\prime \prime}\left(d^{*}\right)\right)^{-1}\left(\begin{array}{l}
0 \\
1
\end{array}\right) \simeq Y \text {. }
\end{aligned}
$$

Since the matrix $\left(F^{\prime \prime}\left(d^{*}\right)\right)^{-1}$ is symmetric, the relation

$$
\frac{\alpha \Xi}{q} X_{y} \simeq \frac{\Xi}{q \alpha} Y
$$


holds. This relation implies the statement of the theorem.

Corollary 2:

Let $d^{*} \in D(F ; \Xi)$. Then the

as $h \rightarrow 0$ and $\varphi \rightarrow 0$.

$$
\Xi F^{\prime \prime}\left(d^{*}\right)^{-1} \simeq\left(\begin{array}{cc}
q \alpha^{-1} X_{x} & q \alpha^{-1} X_{y} \\
q \alpha Y_{x} & q \alpha Y_{y}
\end{array}\right)
$$

Proof follows from the relations on $X$ and $Y$ stated in the proof of Theorem 2.

Theorem 2 may be used if both function $F$ and parameter $\alpha$ are not known but the lattice of points $d(i, j ; F, \Xi)$ are clearly visible. In this case, one can define the vectors $X$ and $Y$ and use Theorem 2 to estimate $\alpha$.

Now we study the aliasing structure when the matrix $F^{\prime \prime}(x, y)$ is invertible at its center $(x, y)$. For each point $d \in D(F, \Xi)$ the number of sets $L\left(n ; G_{d}, \mu\right)$ which intersect with the circle $B_{r}(d)$ is denoted by $\operatorname{Rad}(d, r)$.

Theorem 3: $\quad$ Let $d^{*} \in D(f ; \Xi)$. Then

$$
\lim _{\Xi \rightarrow \infty} \lim _{h \rightarrow 0} \frac{\Xi\left\|F^{\prime \prime}\left(d^{*}\right)\right\||r|^{2}}{2 q \operatorname{Rad}\left(d^{*}, r \sqrt{h}\right)}=1 .
$$

Proof: $\quad$ Let $z=d-d^{*}$. Then

$$
\mu F(z) \simeq H(z)=\frac{\Xi}{2 h}\left(F^{\prime \prime}\left(d^{*}\right) z, z\right)
$$

in the circle $B_{r \sqrt{h}}\left(d^{*}\right)$ as $h \rightarrow 0$. The number of sets $L(n ; H, \mu)$ which intersect with the circle $B_{r \sqrt{h}}\left(d^{*}\right)$ is equal to the integer part of the maximal value of the function $\mu F(z) \simeq \frac{\Xi}{2 h}\left(F^{\prime \prime}\left(d^{*}\right) z, z\right)$ on this circle. Since the matrix $F^{\prime \prime}\left(d^{*}\right)$ is symmetric, its norm is equal to its maximal eigenvalue and the theorem is proved.

Corollary 3: $\quad$ Let $d^{*} \in D(f ; \Xi)$. Then the step $h$ may be approximately defined by the formula

$$
h \simeq \frac{\Xi F^{\prime \prime}\left(d^{*}\right) \||R|^{2}}{2 q \operatorname{Rad}\left(d^{*}, R\right)}
$$

as $h \rightarrow 0$ and $\Xi \rightarrow \infty$.

\section{DISCUSSION}

The results considered above may be useful for describing and explaining the effects which are observed as aliasing structures for bounded values $\Xi>1$. In particular, rather simple modifications of Theorems 1 and 2 may describe the "bleak" aliasing structures which are visible 
in Figures 1 and 2. If $q=2$, then their centers coincide with intersections of the curves $F_{x}^{\prime}(x, y)=i /(\alpha \Xi(2 m+1))$ and $F_{y}^{\prime}(x, y)=j \alpha /(\Xi(2 m+1))$, where $i, j, m \in \mathbb{Z}$.

If $\Xi \gg 1$ then another kind of structure may be observed. The structures described above become very small and they are localized in the small domains in the chaotically painted plane $P$; on the other hand, new regular structures appear which are separated by the chaotic colors. A typical example is displayed in Fig. 3 where the quantized level sets for the function $F_{2}(x, y)=R-\sqrt{R^{2}-x^{2}-y^{2}}$ are painted for $\mu=400000$. Note this kind of structure changes slowly as parameter $\mu$ varies. For some values of the parameter $\mu$ other ("carpet") structures appear. One can see an example for $F_{1}(x, y)=-y^{4}+x^{2} y$ in Figure 4 where $\mu=576000$. The "carpet" structures are very sensitive to the variation of the parameter $\mu$. The strict analysis of quantized pictures for $\Xi \gg 1$ is not yet completed. In particular, the following problems may be of interest for further research.

Problem 1: Construct a mathematical description of aliasing effects for Figures 3 and 4.

Problem 2: Use the asymptotical structures of aliasing pictures for restoring the features of the initial function.

\section{REFERENCES}

[1] A.K. Dewdney, "Wallpaper for the mind: Computer images that are almost, but not quite, repetitive”, Scientific American 9, (1986), pp. 14-23.

[2] A.K. Dewdney, "On fractal mountains, graftal plants and other computer graphics of Pixar", Scientific American 11, (1986), pp. 14-20.

[3] A.E. Lobkovskii, “Mathematical patterns on plane”, Kvant 11, (1987), (in Russian).

[4] T. Pavlidis, "Algorithms for Graphics and Image Processing”, Computer Science Press, 1982.

[5] D.F. Rogers, "Procedural Elements for Computer Graphics", McGraw-Hill, Inc., New York, 1985. 


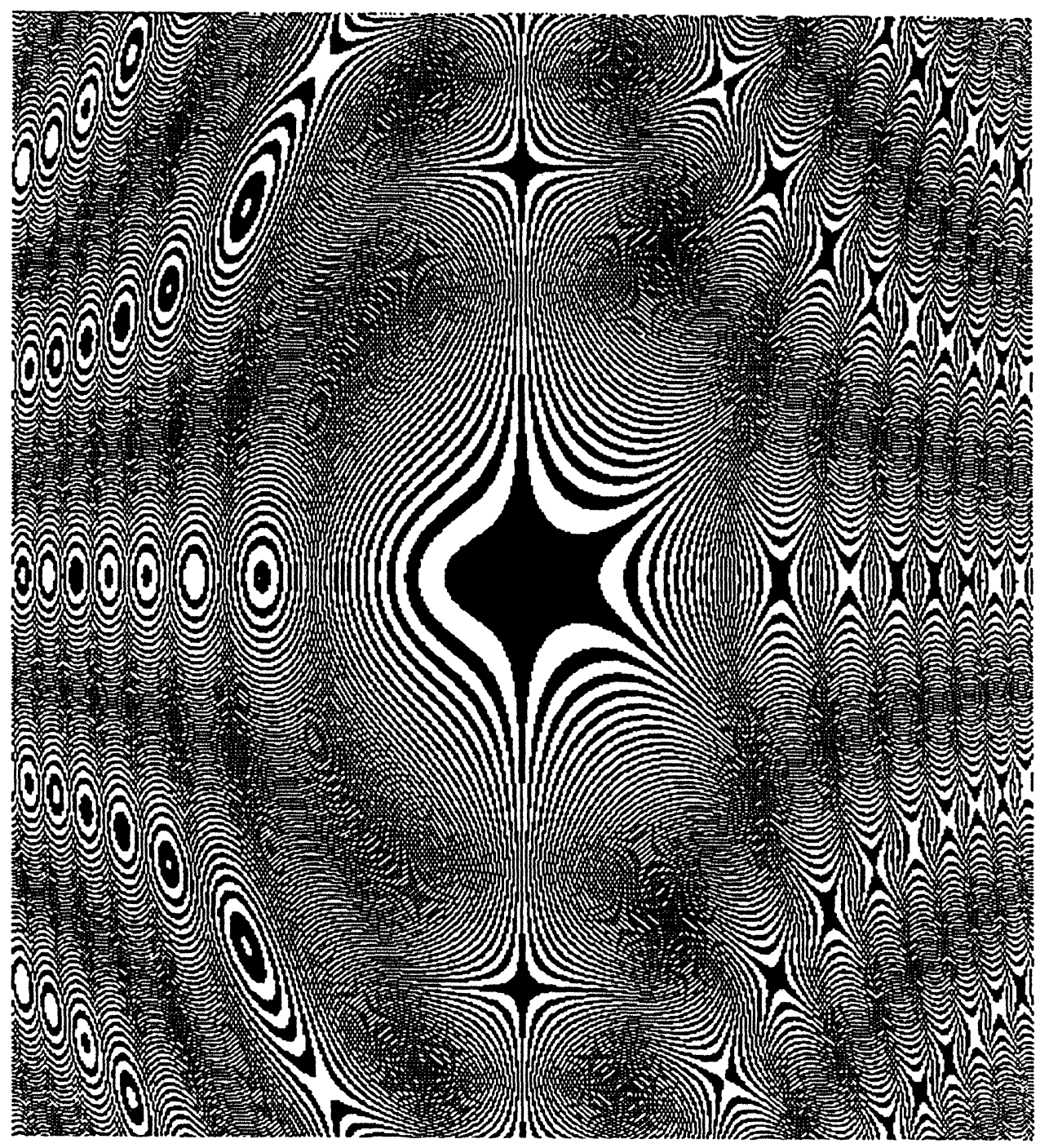

Figure 1 


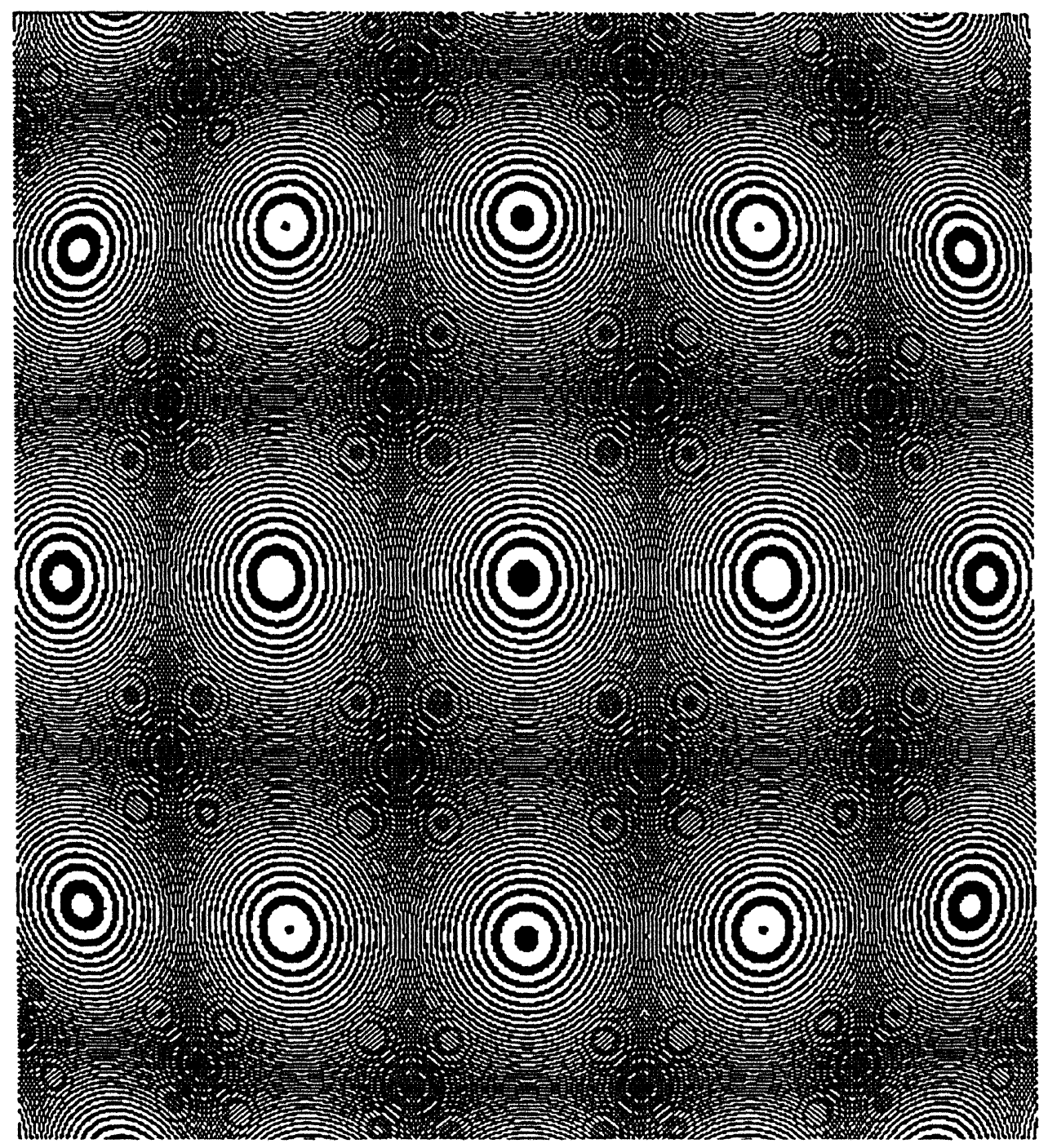

Figure 2 


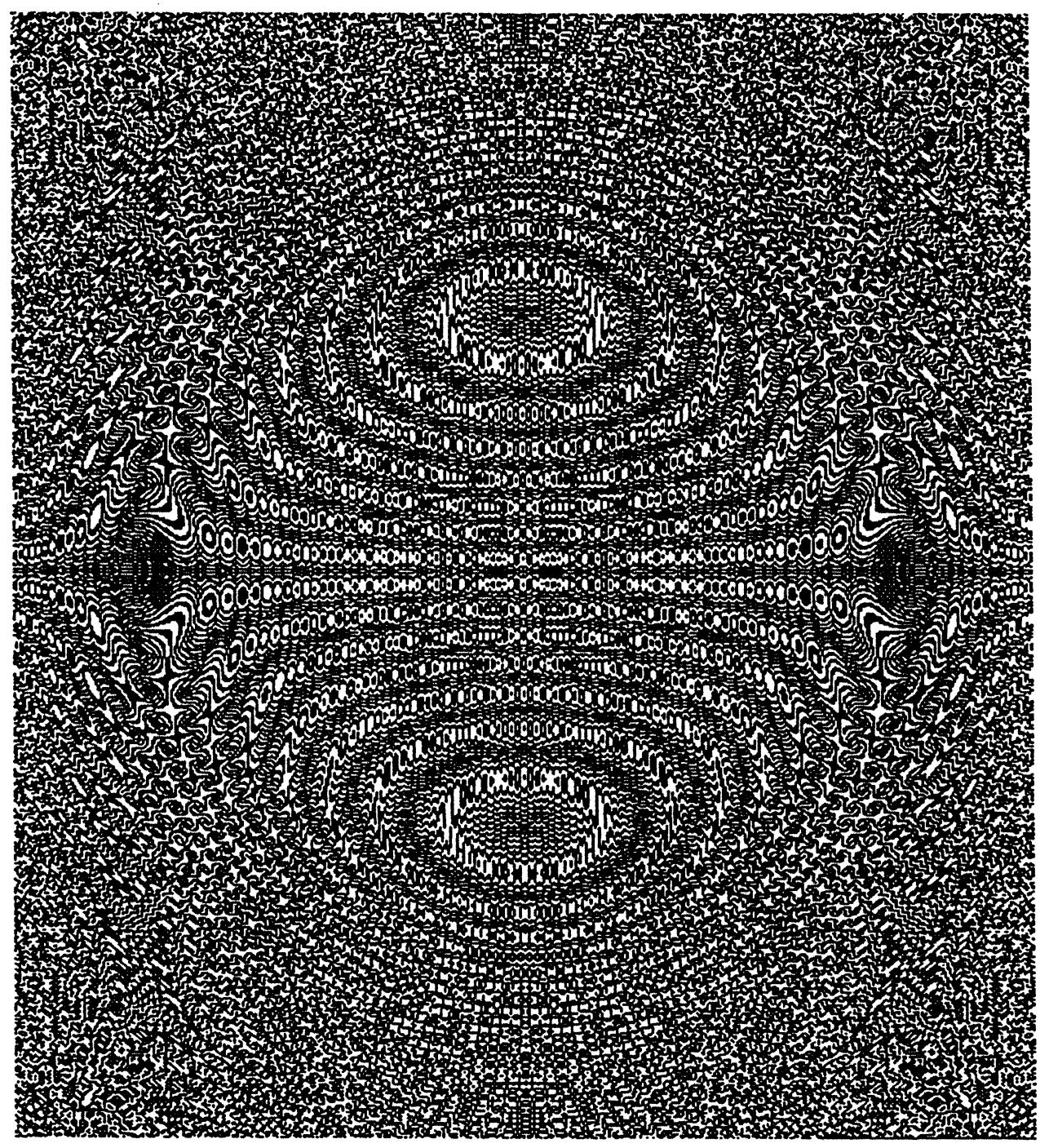

Figure 3 


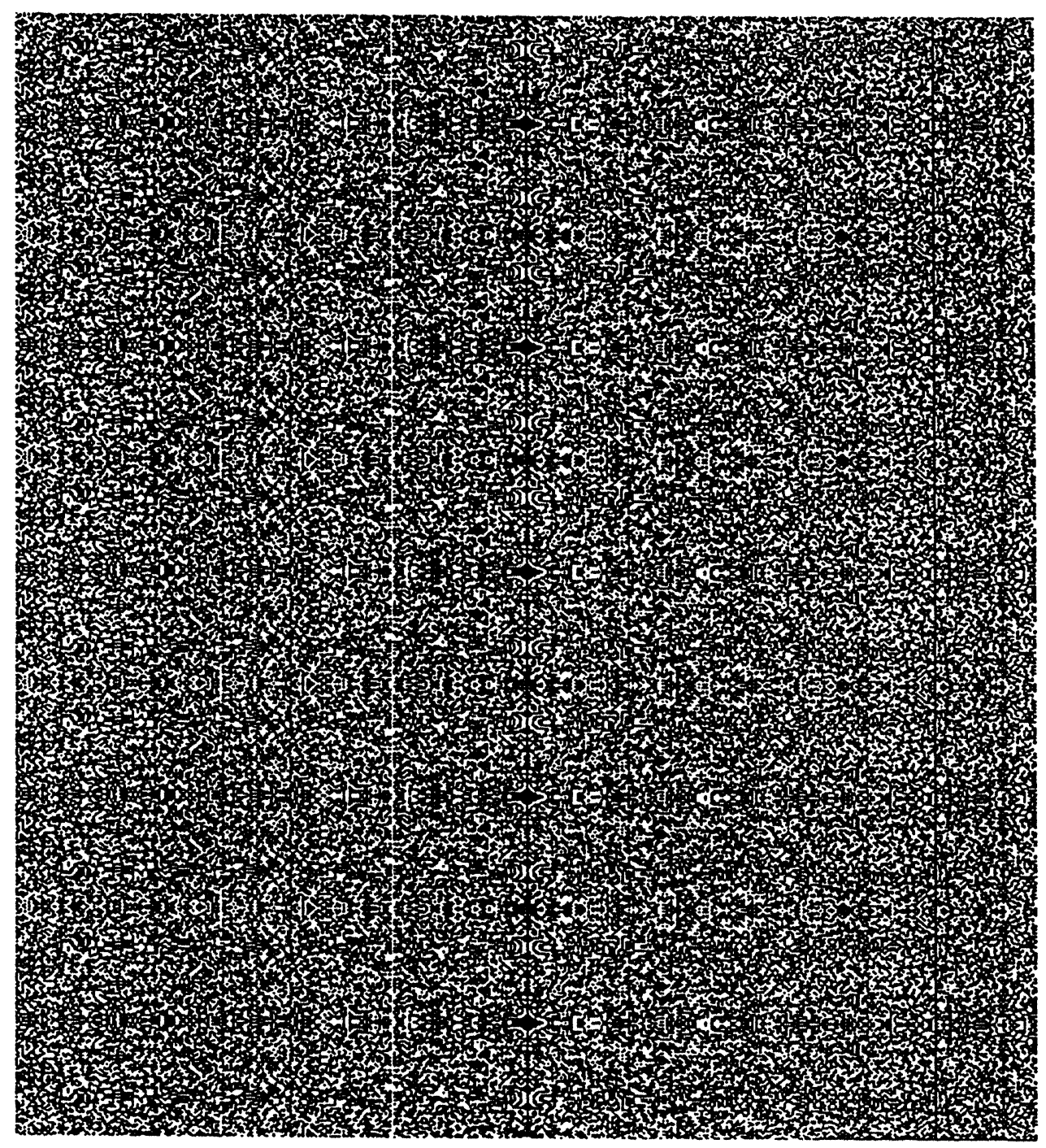

Figure 4 


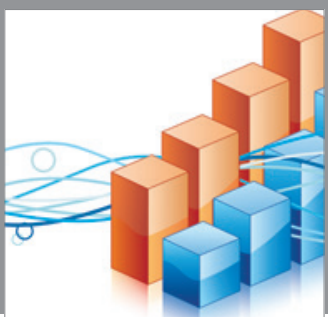

Advances in

Operations Research

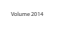

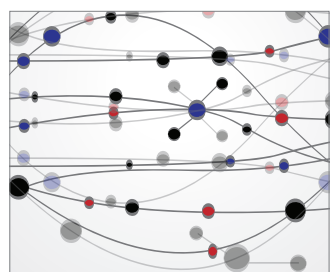

\section{The Scientific} World Journal
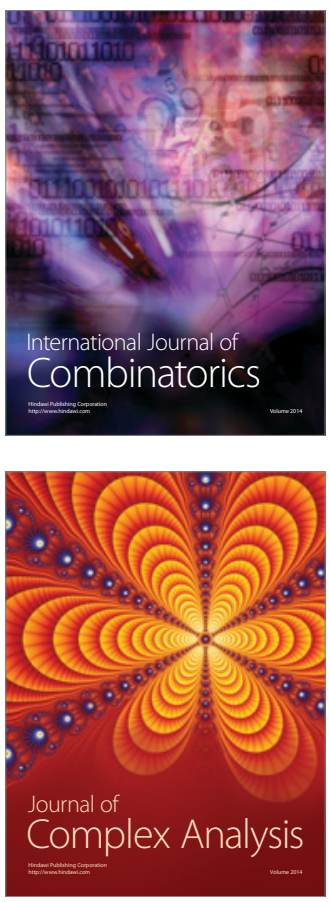

International Journal of

Mathematics and

Mathematical

Sciences
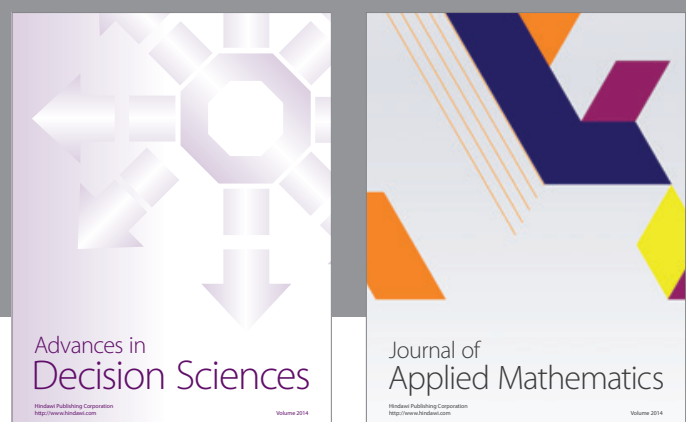

Journal of

Applied Mathematics
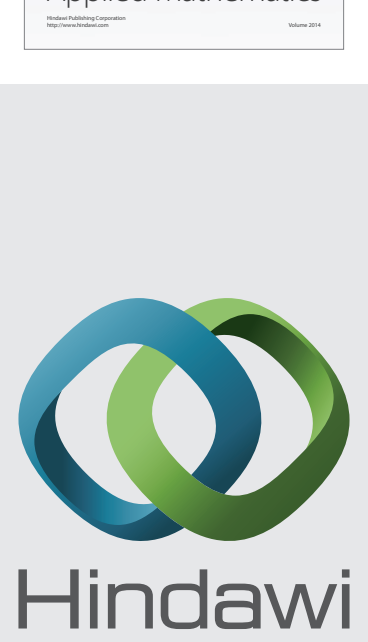

Submit your manuscripts at http://www.hindawi.com
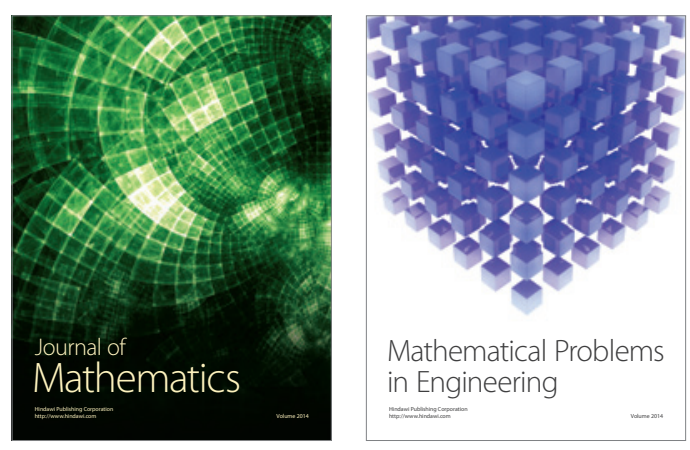

Mathematical Problems in Engineering
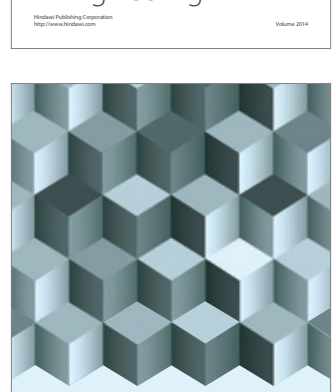

Journal of

Function Spaces
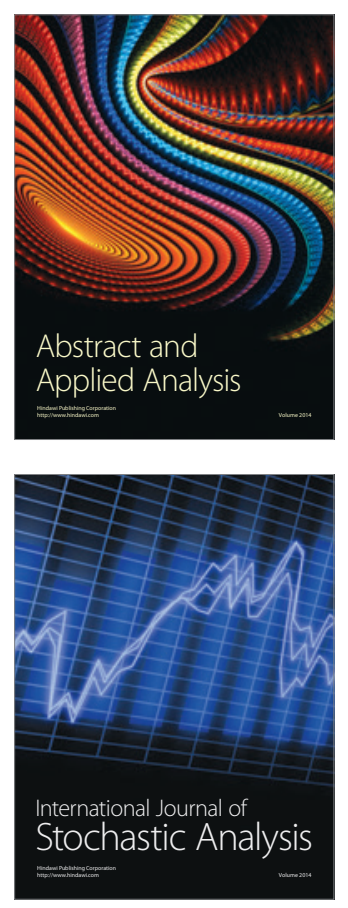

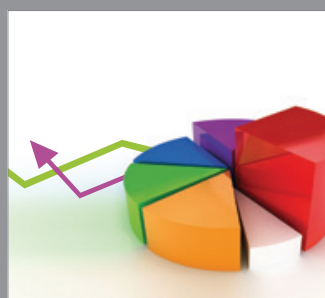

ournal of

Probability and Statistics

Promensencen
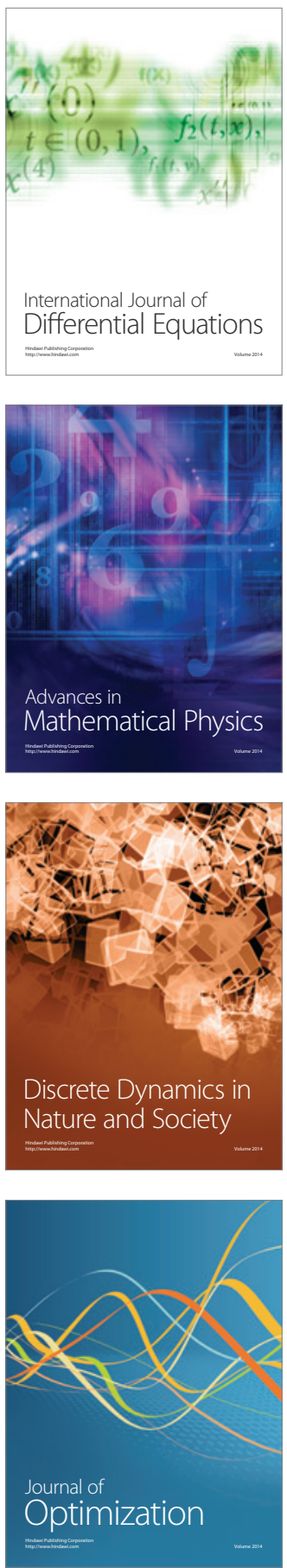